\title{
Application of the Hill Climbing Algorithm to the Geometrical Reconstruction of Vertical Buried Heat Sources Using Vibrothermography
}

\author{
by J. Jaime*, A. Cifuentes*, K. Martínez*, E. Marin*, J. Hernandez-Wong**, A. Mendioroz ${ }^{* * *}$, A. Salazar***
}

*Instituto Politécnico Nacional, (IPN), Centro de Investigación en Ciencia Avanzada y Tecnología Avanzada (CICATA), Unidad Legaria, Legaria 694, Col. Irrigación, C. P. 11500, Ciudad de México, Mexico, jiaimepuldon@gmail.com

${ }^{* *}$ CONACyT, Instituto Politécnico Nacional, (IPN), Centro de Investigación en Ciencia Avanzada y Tecnología Avanzada (CICATA), Unidad Legaria, Legaria 694, Col. Irrigación, C. P. 11500, Ciudad de México, Mexico, hjoel@tetraa.com.mx

*** Departamento de Física Aplicada I, Escuela de Ingeniería de Bilbao, Universidad del País Vasco UPV/EHU, Plaza Ingeniero Torres Quevedo 1, C. P. 48013, Bilbao, Spain, arantza.mendioroz@ehu.eus

\begin{abstract}
The characterization of internal heat sources has been successfully applied in the field of non-destructive testing (e.g. vibrothermography) to obtain the geometrical parameters of vertical cracks by solving the ill-posed inverse problem [1]. In these techniques the crack or defect is heated and the change in the surface temperature is measured, by solving the inverse problem. Metaheuristic algorithms offer an alternative approach for optimization problems. This work aims at exploring the use of the so-called hill climbing metaheuristic algorithm to obtain the geometrical reconstruction of vertical cracks from time resolved vibrothermography measurements.
\end{abstract}

\section{Introduction}

Infrared thermography (IRT) has proven itself as a non-destructive testing (NDT) technique capable of detecting shallow subsurface defects. A major variant of IRT involves optical exciting the sample using light to detect possible defects, such as delaminations and cracks [2]. Different variants have been developed, however these optical techniques are well suited for cracks that offer a strong thermal barrier. In a contact defect no large gap or discontinuity is formed within the specimen and this leads to good thermal contact and makes defect detection and sizing difficult.

A technique that shows promise in this type of defect detection is vibrothermography, both in its lock-in [3] and burst variants [4]. The use of metaheuristic algorithms has not been explored in this area and might offer a potential benefit in computing resources and algorithm complexity. In this work, we explore the use of the hill climbing metaheuristic algorithm and apply it to time resolved (i.e. burst) vibrothermography obtain the geometrical parameters of a buried heat source. This is to be achieved by assuming that the arbitrarily shaped defect can be approximated by a given number of smaller square defects within the heat-source plane. For this purpose, a mesh is created with square elements and it is the algorithms job to find which elements within the mesh contribute to generate the temperature distribution seen at the surface
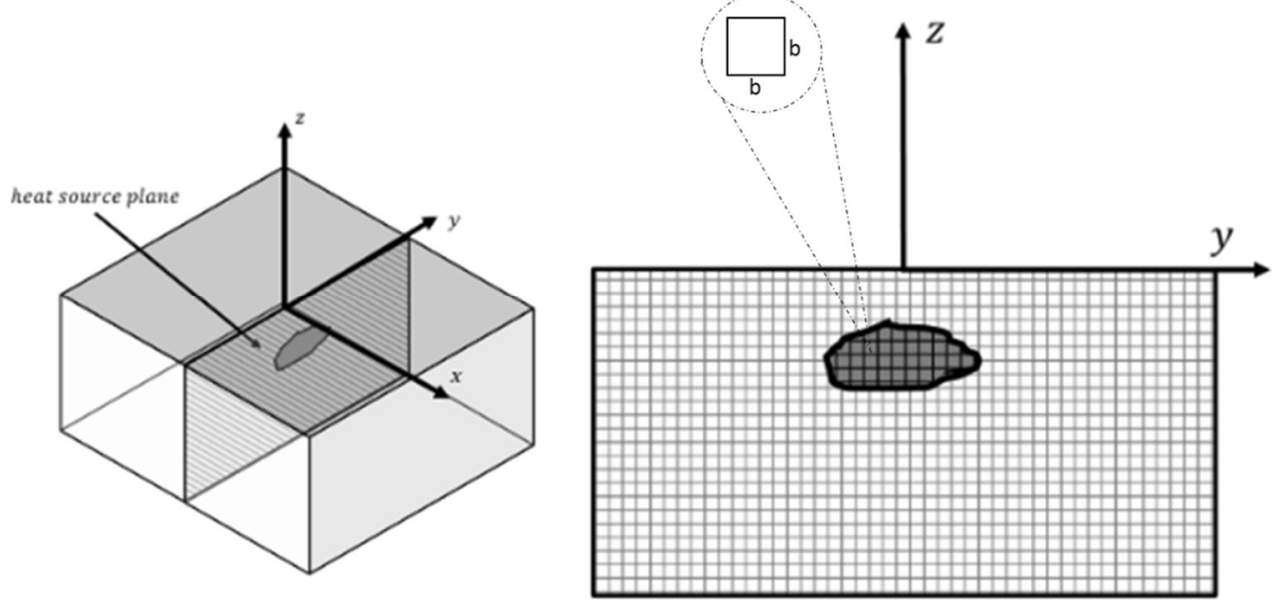

Fig. 1. Problem geometry. 


\section{Mathematical model}

The geometry of a vertical heat source of arbitrary shape is shown in Fig. 1. In the particular case of a buried square heat source of side $b$ located at a depth $d$, the surface temperature is given by [4].

$$
\begin{aligned}
& T(x, y, 0, t)=\frac{I}{2 \pi k} \int_{-b-d}^{-d} \int_{-\frac{b}{2}}^{\frac{b}{2}} \frac{\operatorname{Erfc}\left[\frac{\sqrt{x^{2}+\left(y-y^{\prime}\right)^{2}+z^{\prime 2}}}{\sqrt{4 \alpha t}}\right]}{\sqrt{x^{2}+\left(y-y^{\prime}\right)^{2}+z^{\prime 2}}} d y^{\prime} d z^{\prime} \\
& T(x, y, 0, t)=\frac{I}{2 \pi k} \int_{-b-d}^{-d} \int_{-\frac{b}{2}}^{\frac{b}{2}} \frac{\operatorname{Erfc}\left[\frac{\sqrt{x^{2}+\left(y-y^{\prime}\right)^{2}+z^{\prime 2}}}{\sqrt{4 \alpha t}}\right]}{\sqrt{x^{2}+\left(y-y^{\prime}\right)^{2}+z^{\prime 2}}} d y^{\prime} d z^{\prime}-\int_{-b-d}^{-d} \int_{-\frac{b}{2}}^{\frac{b}{2}} \frac{\operatorname{Erf} c\left[\frac{\sqrt{x^{2}+\left(y-y^{\prime}\right)^{2}+z^{\prime 2}}}{\sqrt{4 \alpha(t-\tau)}}\right]}{\sqrt{x^{2}+\left(y-y^{\prime}\right)^{2}+z^{\prime 2}}} d y^{\prime} d z^{\prime} \quad \mathrm{t}>\tau
\end{aligned}
$$

where $\alpha$ is the thermal diffusivity, $k$ is the thermal conductivity and $\tau$ is the time of excitation, i.e. burst duration.

Utilizing the superposition principle, it is possible to obtain the surface temperature for any combination of mesh elements. It is the algorithm's task to determine the correct combination of elements to approximate the surface temperature generated by a given defect.

\section{Metaheuristic algorithms}

Optimization consists of finding the best possible solution to a given problem, for the purpose of either maximizing or minimizing a specific objective function. The simplest variant of optimization represents a problem where there are no restrictions and if they exist they are of equality, with less or equal number of variables that the objective function[5].

Formally, an optimization problem can be defined as follows[6]:

Given the search space $\Omega \subseteq \mathrm{R}^{\mathrm{D}}$, the objective function $f: \Omega \rightarrow \mathrm{R}$ and the comparison relation $\geqslant \in\{\leq ; \geq\}$, the resolution of an optimization problem is to find the set $\mathrm{X}$ of optimum solutions defined as:

$$
X=\left\{x^{*} \in \Omega \mid f\left(x^{*}\right) \geqslant f(x) ; \forall x \in \Omega\right\}
$$

When $\geqslant$ is represented by the relation $\leq$ then the problem is minimization, otherwise maximization.

The existence of a large number and variety of difficult problems, which appear in practice and need to be solved efficiently, impels the development of procedures capable of reaching good states or solutions, even if they are not optimal[5]. These methods, in which the speed of the process is as important as the quality of the solution obtained, are called heuristic or approximate[6]. The heuristics are tailor-made and designed to solve a specific problem and / or instance, which is why metaheuristics arise. They are general-purpose algorithms that can be applied to solve any optimization problem [7]. It is also important to note that according to the non-free lunch theorem proposed by Wolpert and Macready [8], there is no optimization algorithm whose performance is superior to any other over all possible optimization functions. Therefore, depending on the problem we must analyze the operation of the algorithms.

A wide variety of metaheuristics has been developed using different criteria, such as the use or not of memory, deterministic or stochastic, inspired by the evolution of nature, among others. One of the well-known metaheuristics method is The Hill Climbing algorithm.

\section{The Hill Climbing Metaheuristic Algorithm}

The Hill Climbing $(\mathrm{HC})$ algorithm [9] has its inspiration in an analogy with an imaginary situation in which a climber seeks to climb the summit of a mountain. Due to the fog, the mountaineer only knows if the immediate surface to him is ascending or descending. The approach is focused to maximize; but the algorithm is also valid for minimizing. These algorithms are general purpose methods and their main limitation is the convergence to the nearest local minimum. Therefore, if the evaluation of the candidate solution, $X_{C}$, is no better than that of the current solution $X_{b e s t}$, it indicates that the current solution is a local minimum and the $\mathrm{HC}$ will never leave this point; different strategies exist to avoid this problem and must be taken into account. The size of the neighborhood has been shown to significantly influence the behavior of the $\mathrm{HC}$, since more solutions are neighboring the current, there will be less probability of falling into a local minimum. Another interesting point in the improvement of the algorithm is achieved by accepting candidate solutions whose evaluation is the same as the current one[10].The scheme of the general operation of the HC is described in figure 2. 


\section{Algorithm I: Pseudocode of the Classic Hill Climbing algorithm}

1: Input: N (Maximum number of iterations), $\mathrm{T}$ (Size of the neighborhood of the current state)

2: Output: $\mathrm{x}_{\text {best }}$ (Best solution found)

3: $\mathrm{x}_{\text {best }} \leftarrow$ GeneratelnitialSolution ()

4: repeat

5: $x_{c} \leftarrow$ BestNeighbor (xbest, $T$ )

6: if $f\left(x_{c}\right)<f\left(x_{\text {best }}\right)$ then

7: $\mathrm{x}_{\text {best }} \leftarrow \mathrm{x}_{\mathrm{c}}$

8: end if

9: $\mathrm{n}=\mathrm{n}+1$

10: until stop criterion is reached, $n>N$

11: return $\mathrm{x}_{\text {best }}$

Fig. 2. Scheme of the general operation of the HC algorithm[5].

\section{Results and Conclusions}

The HC algorithm has shown great promise in finding hidden defects. Shown in Fig. 3 is the reconstruction of three buried synthetic defects at three different depths with a $5 \%$ amplitude noise level and without noise. The best five solutions obtained by the $\mathrm{HC}$ algorithm are shown superimposed with the defect being shown in red. The algorithm correctly approximates the defect, further iterations can produce an improved result. Finally, the $\mathrm{HC}$ algorithm has been applied to vibrothermography experimental data on metallic samples containing calibrated buried heat sources.

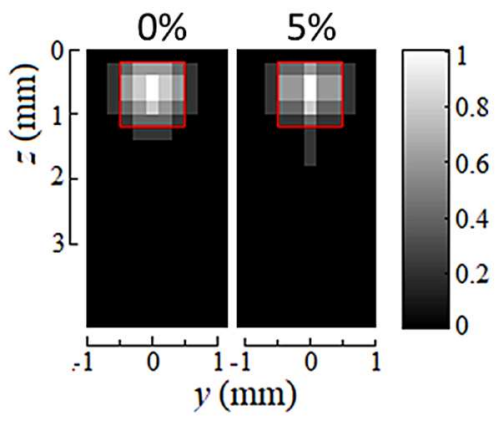

a)

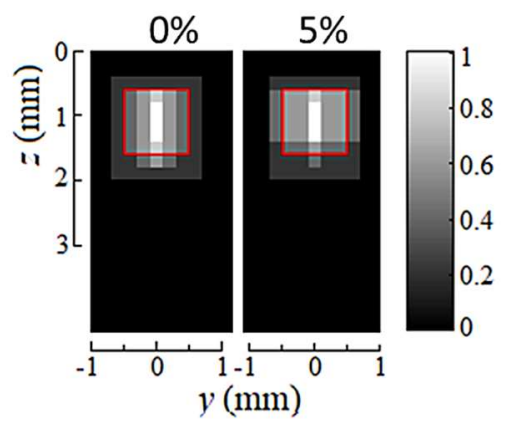

b)

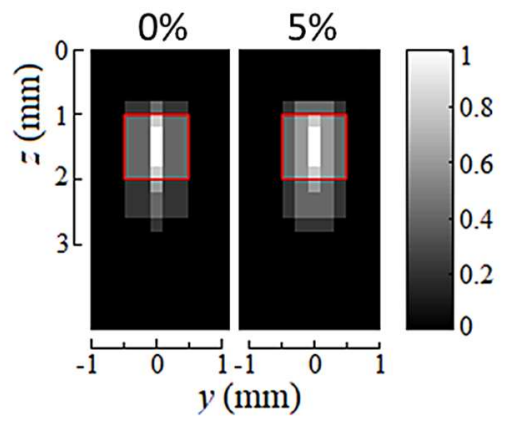

c)

Fig. 3. Reconstruction of a set of synthetically generated buried $1 \mathrm{~mm} \times 1 \mathrm{~mm}$ defects using the HC algorithm for $0 \%$ and $5 \%$ amplitude noise levels. a) $200 \mu \mathrm{m}$, b) $600 \mu \mathrm{m}$ and c) $1 \mathrm{~mm}$. Mesh size is $200 \mu \mathrm{m} \times 200 \mu \mathrm{m}$.

\section{Acknowledgments}

The authors would like to acknowledge financial support by Ministerio de Economía y Competitividad (DPI201677719-R, AEI/FEDER, UE), by Universidad del País Vasco UPV/EHU (GIU16/33), SIP-IPN (Research Grants 20160144, 20170545) and CONACyT (2015-02-1042, 264093, 2016-01-2482). The support of COFAA-IPN by the SIBE and BEIFI programs is also acknowledged. 


\section{REFERENCES}

[1] R. Celorrio, A. Mendioroz, and A. Salazar, "Characterization of vertical buried defects using lock-in vibrothermography: II. Inverse problem," Measurement Science and Technology, vol. 24, p. 065602, 2013.

[2] X. P. Maldague, Nondestructive evaluation of materials by infrared thermography: Springer Science \& Business Media, 2012.

[3] A. Salazar, A. Mendioroz, E. Apiñaniz, et al., "Characterization of delaminations by lock-in vibrothermography," in Journal of Physics: Conference Series, 2010, p. 012079.

[4] A. Mendioroz, R. Celorrio, A. Cifuentes, et al., "Sizing vertical cracks using burst vibrothermography," NDT \& E International, vol. 84, pp. 36-46, 2016.

[5] Doerner, K., Metaheuristics: Progress in complex systems optimization. Springer Verlag, 2007. 39.

[6] Roli, C.B.a.A., Metaheuristics in combinatorial optimization: Overview and conceptual comparison. ACM Computing Surveys, 2003. 35(3): p. 268-308.

[7] Birattari, M., Tuning Metaheuristics: A Machine Learning Perspective. Studies in Computational Intelligence. Springer, 2005.

[8] Macready., D.H.W.a.W.G., No free lunch theorems for search. 1995.

[9] E. H. Aarts and J. K. Lenstra, Local Search in Combinatorial Optimization: Princeton University Press, 1997.

[10] R. Greiner, "Probabilistic hill-climbing.Theory and applications," in Conference-Canadian Society of Computational Studies of Intelligence (CSCSI-92), 1992, pp. 60-67. 